\title{
Real-time Assembly of Coordination Patterns in Human Infants
}

Ori Ossmy and Karen E. Adolph

Department of Psychology and Center for Neural Science, New York University, New York, NY, USA 


\section{Summary}

Flexibility and generativity are fundamental aspects of functional behavior that begin in infancy and improve with experience. How do infants learn to tailor their real-time solutions to variations in local conditions? On a nativist view, the developmental process begins with innate prescribed solutions and experience elaborates on those solutions to suit variations in the body and the environment. On an emergentist view, infants begin by generating a variety of strategies indiscriminately and experience teaches them to select solutions tailored to the current relations between their body and the environment. To disentangle these accounts, we observed coordination patterns in 11-month-old pre-walking infants with a range of cruising (moving sideways in an upright posture while holding onto a support) and crawling experience as they cruised over variable distances between two handrails they held for support. We identified infants' coordination patterns using a novel combination of computer-vision, machine-learning, and time-series analyses. As predicted by the emergentist view, the least experienced infants generated multiple coordination patterns inconsistently regardless of body size and handrail distance, whereas the most experienced infants tailored their coordination patterns to bodyenvironment relations and switched solutions only when necessary. Moreover, the beneficial effects of experience were specific to cruising and not crawling, although both skills involve antiphase coordination among the four limbs. Thus, findings support an emergentist view and suggest that everyday experience with the target skill may promote "learning to learn," where infants learn to assemble the appropriate solution for new problems on the fly.

Keywords: infants, artificial intelligence, machine learning, locomotion, motor development 


\section{Introduction}

Flexibility and generativity are what make skills truly functional. Consider human walking: In skilled walkers, interlimb coordination follows a universal pattern where each leg moves the same distance with the same timing, in precise anti-phase with the other leg [1]—but only while stepping on a treadmill or walking in a straight path over uniform ground. In a real-world environment, coordination cannot follow a prescribed formula. Instead, the distance, timing, and direction of leg movements must be continually modified to suit changes in local conditions [2, 3]. Moreover, sometimes all four limbs contribute to real-time solutions (e.g., crawling) and sometimes, environmental supports must be incorporated into the solution (e.g., rock climbing). Such flexibility and generativity begin in infancy and improve with experience (for reviews, see $[4,5,6])$. For example, with experience, infants learn to modify their step length, speed, and interlimb phasing to keep balance while walking over slopes, drop-offs, and bridges, and they learn to plan gait modifications ahead of time based on visual information from a distance [7].

Experience-related improvements raise an important developmental question—how do infants learn to adapt their coordination patterns to variations in local conditions? One possibility is that the developmental process begins with innate, neuromuscular mechanisms that generate prescribed coordination patterns for moving the two legs in walking and all four limbs in crawling [8-12]. On this nativist view, experience elaborates on the basic coordination patterns with more adaptive, real-time solutions to cope with variations in the body and environment. In contrast, on an emergentist view, infants begin with a proliferation of variable coordination patterns, and experience teaches infants to select real-time solutions attuned to variations in bodyenvironment relations [13-16]. That is, coordination patterns are emergent, and experience teaches infants to assemble the appropriate solution on the fly. However, existing evidence cannot distinguish between innate versus emergent coordination patterns because both camps study infants moving over uniform ground or stepping on a motorized treadmill and thus predict the same outcome-converging to more consistent coordination patterns with experience. 
What's needed are manipulations of the environment where predictions of the two accounts differ.

Here, we investigated the role of experience in infants' acquisition of behavioral flexibility by focusing on "cruising"-when pre-walking infants step sideways using an environmental support (coffee table, couch, etc.) to keep balance. Cruising is a brief, transitory skill that overlaps with crawling and disappears after infants can walk $[17,18]$. Like walking, cruising involves an upright posture, and like crawling, cruising involves movements of all four limbs; indeed, cruising may be considered functionally as "upright crawling" [17]. Crawling experience leads to a consistent anti-phase pattern where diagonal limbs (right arm, left leg, etc.) move together $[10,19,20]$, but effects of experience on interlimb coordination in cruising are unclear $[21,22]$. Moreover, unique to cruising, infants must incorporate environmental support into their coordination patterns [21], and the nature of the support (distance between furniture, height of the table, compliance of the couch, etc.) influences which patterns are viable $[17,23]$.

In two experiments, we used computer-vision, machine-learning, and time-series analyses $[24,25]$ to test the role of experience in interlimb coordination based on videos of cruising infants. In Experiment 1, we observed infants under uniform conditions (infants cruised over a continuous handrail) to test effects of experience on cruising proficiency and variability in coordination patterns. In Experiment 2, we observed infants under varying conditions (infants cruised over two handrails separated by different distances) to test effects of experience on interlimb coordination. If the nativist view is correct-that infants first master a specific solution and then expand it to other situations-we should see use of the same coordination pattern in novice cruisers (regardless of handrail distance) but additional patterns in experienced cruisers (tailored to handrail distance). However, if the emergentist view is correct-that infants shift from trying different patterns inconsistently to adapting interlimb coordination to the constraints of current body-environment relations - then both novice and experienced cruisers should use 
multiple coordination patterns, but only experienced cruisers should tailor interlimb coordination to changes in handrail distance.

\section{Results}

\section{Infants Learn to Cruise Faster with the Same Number of Limb Movements}

In Experiment 1, infants cruised repeatedly over a 302-cm long, wooden handrail $(N=$ 24; Figure $1 \mathrm{~A}$ and STAR Methods). We held age constant (11 months \pm 1 week) and allowed cruising (13-103 days) and crawling experience (29-119 days) to vary. Most infants began crawling at younger ages than cruising (Figure S1A), but crawling and cruising experience were not correlated, $r(22)=-.10, p=.64$ (Figure 1B).

First, we tested effects of cruising and crawling experience on overall speed and the number of arm and leg steps (see STAR Methods). Step number reflects the average size of limb movements (fewer steps implies larger movements). Although step number and speed varied widely across infants, only speed was correlated with cruising experience, $r(22)=.65$, $p<.00$, indicating that practice cruising led infants to move faster (Figure $1 \mathrm{C}$ ), not to take larger steps (Figure S2A-B). Crawling experience was unrelated to step number or speed, $p s>.14$ (Figure S2C-E), suggesting that practice moving all four limbs during crawling did not improve cruising skill.

\section{Cruising Experience Predicts More Efficient Interlimb Coordination in Real Time}

Infants did not improve due to the total number of limb movements. Therefore, we examined their interlimb coordination, that is, how infants changed the distance between their arms and between their legs from moment to moment. We used computer vision to represent coordination as a movement time series (MTS); see Figure 2A-C and STAR Methods. Figure 2D shows exemplars of arm and leg MTS in one representative trial of the most and least experienced cruiser (see Figure S3 for MTS of all infants). Positive z-scores in the time series indicate increased distance between the arms or the legs, and negative z-scores indicate 
decreased distance. Figure $2 \mathrm{E}$ shows three cycles of a common coordination pattern in the MTS (bottom panel) and the infant's movements from the relevant video frame (top panel).

To test the consistency of infants' coordination patterns, we quantified variability in arm and leg movements independently (see STAR Methods). If the coordination pattern is stable, variability is low; and if the coordination pattern is inconsistent, variability is high. Infants with more cruising experience showed less variability in arm steps, $r(22)=-.46, p<.03$, and leg steps, $r(22)=-.41, p<.05$, but crawling experience was not correlated with movement variability (Figure 3A-B). Moreover, arm and leg variability were negatively correlated with cruising speed, $r(22)=-$ .73 and $r(22)=-.75$ respectively, $p s<.00$ (Figure S4A-B). Thus, experienced cruisers moved their arms and legs faster than novice cruisers, and they did so more consistently from step to step.

Experienced cruisers were also better at coordinating arm and leg movements in real time. We quantified the real-time coordination between arm and leg MTS in three ways. First, we tested similarity by calculating the cross-correlation between the arm and leg MTS (see STAR Methods). Figure 3C shows that similarity in the two MTS increased with cruising experience, $r(22)=.53, p<.01$, but not with crawling experience. Increased similarity was accompanied by increased cruising speed, $r(22)=-.4, p<.05$ (Figure S4C).

Second, we analyzed the coordination between arm and leg MTS across the video frames in each trial using Pearson correlations (see STAR Methods). A large positive correlation indicates a precise in-phase strategy in which infants open their arms and legs on the same side of the body simultaneously and then close their arms and legs on the other side of the body simultaneously, as in a pace-like gait; conversely, a large negative correlation indicates a precise anti-phase strategy in which infants open their legs and close their arms at the same time and then close their legs and open their arms at the same time in an oscillatory manner, as in a trot-like gait (see exemplar plots in Figure 2D). Every infant showed negative correlations (range $=-.12$ to $-.64, M=-.36$ ), indicating a common anti-phase strategy. Moreover, infants with more cruising experience showed larger negative correlations between the two MTS than 
infants with less cruising experience, $r(22)=-.42, p<.04$, but crawling experience was unrelated (Figure 3D). As with other measures, faster cruising speed was associated with larger correlations, $r(22)=-.59, p<.00$ (Figure S4D). Thus, with cruising experience, infants learned an efficient real-time solution in which they alternated arm and leg movements for fast cruising.

Finally, we calculated the real-time phase-locking value between arm and leg MTS (see STAR Methods). A high phase-locking value indicates that the lag between oscillatory arm and leg movements is constant over time. Higher phase-locking values were positively correlated with cruising experience, $r(22)=.41, p<.05$, but not correlated with crawling experience (Figure 3E). As with other coordination measures, phase-locking values were correlated with cruising speed, $r(22)=-.40, p<.05$ (Figure S4E). Thus, leg-arm coordination in experienced cruisers was consistent across the entire trial.

In contrast to cruising experience and speed, infants' body dimensions (height, weight, leg length, and wingspan from fingertip to fingertip) were not correlated with measures of movement or coordination ( $r s<.37, p s>.07$; Table $S 1$ ), suggesting that improvements were not due to physical characteristics such as longer arms and legs.

\section{Infants Adapt Patterns of Coordination to Cope with Distance between Handrails}

What happens, however, when the environment is not constant? Variations in the environment are endemic in everyday locomotion. For cruising infants, the distance between support surfaces varies (couch to coffee table, bed to nightstand, etc.). In such cases, do cruising infants rely on their common anti-phase coordination pattern or do they create new solutions on the fly? The nativist view predicts that novice cruisers will use the trot-like gait evidenced in a uniform environment (as in Experiment 1) across variations in the environment, whereas experienced cruisers will use additional patterns. The emergentist view predicts that both novice cruisers and experienced cruisers will use a variety of coordination strategies, but only experienced cruisers will tailor their solutions to body-environment relations. 
In Experiment 2, we tested a new sample of 11 -month-olds ( \pm 1 week) cruising over variable distances between two handrails they held for support $(N=22$; see Figure $4 \mathrm{~A}$ and STAR Methods). As in Experiment 1, infants had a wide range of cruising (6-122 days) and crawling experience (14-170 days); experience with the two forms of locomotion was related, $r(20)=.40, p=.06$ (Figure 4B and Figure S1B). Sessions began with two trials with the handrails abutting (i.e., infants cruised over a solid handrail as in Experiment 1). Then we separated the handrails. Smaller distances between handrails were easily within infants' arm reach, and larger distances required a big stretch, but all distances required planning to prevent the hands from falling into the gap and the legs from getting too far away from the hands. Because our focus was on interlimb coordination, we only analyzed trials (331 in total) in which infants cruised successfully across the handrail distance (see STAR Methods and Supplemental Information for details about variations in distance and the criteria for successful cruising).

After verifying that infants in Experiment 2 cruised over a continuous handrail using the same anti-phase strategy as in Experiment 1 (see Supplemental Information for replication), we analyzed trials where infants cruised over varying gaps between handrails using Pearson correlations between arm and leg distance across video frames. In contrast to the continuous handrail, infants showed no evidence of a consistent anti-phase strategy: The correlations were both positive and negative ( $M=-.13$; range across trials: -.61 to .39$)$, with negative correlations in only $37 \%$ of the trials. Moreover, visual inspection of the videos confirmed that infants did not perform oscillatory movements to cross the distance between handrails. Thus, we created a different type of MTS based on the sequence and timing of all four limb movements, rather than arms and legs separately. Because trials varied in duration, we used dynamic time warping (DTW) to calculate a similarity index for each pair of trials (see STAR Methods). High similarity between the two MTS indicates a similar real-time strategy. Thus, the procedure allowed us to compare similarity across trials. Figure 5A depicts the MTS in three trials (positive numbers denote increased distance, and negative numbers denote decreased distance). The top two 
trials show a similar strategy: Infants moved the leading arm and leg at the same time (green and purple curves increased together in both MTS), then brought the trailing arm to the leading arm to close the distance (green curves decreased), and then brought the trailing leg near to the leading leg (purple curves decreased). These top two trials differ from the bottom trial in which the leading leg moved to increase the distance between legs (purple curve increased), the leading arm moved before the leg stopped moving (green curve increased), then the trailing leg closed the distance (purple curve decreased), and finally the trailing arm moved to the leading arm (green curve decreased). Thus, the top two MTS are more similar to each other than to the MTS in the bottom trial.

Comparing the similarity of each pair of trials across all infants and trials yielded a movement similarity matrix where the value of each cell reflects the degree to which the pair of trials is similar. Thus, the similarity matrix encapsulates essential information about infants' realtime strategies, which allowed us to cluster trials based on their similarity. We used a densitybased cluster analysis that makes no assumptions about the number of strategies (Figure 5B; see STAR Methods). If trials sort into more than one cluster, that would provide evidence for distinct strategies.

The cluster analysis revealed eight real-time strategies, which we validated with visual inspection of the video for each trial. Figure 6 illustrates the primary components of each strategy. Strategies differed based on the temporal order in which infants moved their limbsthat is, how infants moved their leading and trailing arms/legs when they started and finished crossing the distance between handrails. Infants used four ways to begin crossing. They either stretched their leading leg without moving their arms (strategies \#1 and \#2), stretched their leading leg and leading arm simultaneously (strategy \#3), stretched their arms without moving their legs (strategy \#7), or stretched their leading arm and trailing leg simultaneously (strategies $\# 4, \# 5, \# 6$, and \#8). On the second handrail, at the end of the trial, infants always moved their trailing arm to complete crossing, but strategies differed in what infants did with the other limbs. 
At the same time that they moved their trailing arm, infants either moved their trailing leg (strategies \#1, \#3, and \#4), moved their leading leg (strategies \#2, \#7, and \#8), moved both leading leg and leading arm (strategy \#5), or did not move other limbs at all (strategy \#6).

Strategies differed at different handrail distances within and across infants (Figure S5). Strategy \#1 was most common, used by all infants (Figure 6, bottom-right panel), occurring at most distances (23 out of 25; Figure S5). Each real-time strategy was used by at least six infants, and all infants used 3-6 different strategies (Figure 6, bottom-right panel), indicating that all infants used multiple strategies, unlike Experiment 1 where infants used the single anti-phase strategy. Moreover, infants used the anti-phase coordination pattern only in strategy \#8. The correlation between arms and legs for the anti-phase strategy was $M=-.47(S D=.37)$, whereas for other strategies, correlations ranged from -.25 to .10 .

\section{Infants Learn to Use the Appropriate Strategy at the Right Time}

We tested whether the use of different strategies changed according to changes in bodyenvironment relations (i.e., ratio between infants' wingspan and handrail distance). Trials with different ratios require different real-time strategies. For example, a high ratio indicates a more challenging task and requires a strategy in which infants stretch their arms as wide as they can while maintaining balance. With a low ratio, there is no need for maximum arm stretching, and infants can use the same anti-phase strategy they used when the handrail was continuous. Figure 7A shows that real-time strategies were indeed geared to the body-environment ratio: strategy \#1 for high ratios > 0.5; strategies \#2-5 for moderate ratios >0.25 and < 0.5; and strategies \#6-8 for low ratios < 0.25. Finally, we examined the use of high-, moderate-, and lowratio strategies as a function of cruising experience. To that end, we performed a median split based on cruising experience $\left(M_{\text {experienced }}=84.91 \pm \mathrm{SD}=23.11\right.$ cruising days, $M_{\text {Novice }}=$ $32.45 \pm 15.92 ; t(10)=5.16, p<.001)$. Experienced cruisers used the appropriate strategy more consistently than novice cruisers (diagonals in Figure 7B). 


\section{Infants Learn to Switch Strategies Only When Necessary}

Switching strategies is not always necessary. If the body-environment relation is constant, modifying the coordination pattern indicates inconsistency in strategy selection. To test individual differences in strategy switching, we defined a switching index $S$ as the number of real-time strategies for different handrail distances. Because adjacent distances are similar (e.g., 28 and $30 \mathrm{~cm}$ ), we systematically varied the window size for calculating $S$ (see STAR Methods). When $S$ equals 1 , switching is most consistent-the same real-time strategy is used for similar distances, suggesting that infants switched real-time strategies only when required. Larger S values indicate inconsistent switching, that is, different real-time strategies were used for similar distances. We found that the $S$ index was negatively correlated with cruising experience across relatively small window sizes (Figure $7 C$ ); $r(20)=-.94, p<.001$ for the smallest $2-\mathrm{cm}$ window; $r(20)=-.79, p<.001$ for $4 \mathrm{~cm}$; and $r(20)=-.55, p<.008$ for $8 \mathrm{~cm}$. In contrast, for relatively large window sizes $(12$ and $16 \mathrm{~cm}$ ), we did not find significant correlations, $r s(20)>-.38, p s>.07$, meaning that switching was specific to the wingspandistance ratio. Decrease in the switching index with cruising experience indicates that experienced cruisers were more "consistent switchers" than novice cruisers. Although the $S$ index decreased with crawling experience, we did not find significant correlations with crawling experience for any window size (Figure 7D), ps $>.05$. This result shows that improvement in switching strategies does not result from overall experience moving the four limbs, but rather specific experience with the relevant skill.

\section{Discussion}

We used cruising as a model system to understand how infants learn to adapt their actions to changes in local conditions. When cruising, infants must incorporate environmental support into their coordination patterns. With a uniform handrail, infants can use a single coordination pattern, but variable distances between two handrails requires infants to modify their movements according to the changing relations between their body size (wingspan) and 
handrail distance. As predicted by both nativist and emergentist accounts of coordination patterns in a uniform environment, we found that infants cruised faster and more consistently with cruising experience (Experiment 1). However, infants' real-time strategies in a variable environment were consistent only with the emergentist account: As predicted, experienced cruisers used solutions tailored to the body-distance ratio, whereas novice cruisers changed solutions willy-nilly regardless of distance or body size (Experiment 2).

\section{Locomotor Solutions as Real-Time Coordination Patterns}

Previously, researchers assessed infants' solutions to locomotor problems based on manual video coding of the form of locomotion [e.g., cruising, crawling, scooting, and so on, as in 17] or variations in posture within a form of locomotion [e.g., using a handrail to walk in "hunchback," "windsurfing," or "mountain climbing" postures as in 26]. With adults, researchers can assess interlimb coordination by attaching motion sensors to the body [27] . However, motion sensors disrupt infants' natural activity. Thus, we developed a new, video-based, sensorfree approach that allowed us to quantify infants' real-time coordination patterns in both a uniform and variable environment. Our analytic approach builds on similarity analysis in math, psychology, and neuroscience [28-30], and recent advances in artificial intelligence and computer vision [24, 31, 32]. It provides precise, quantitative, and objective details about infants' coordination patterns from moment to moment and facilitates deeper understanding of real-time motor solutions.

\section{How Experience Affects Real-Time Coordination Patterns}

Under uniform conditions (continuous handrail) in Experiment 1, experience facilitated cruising speed as infants converged to a stable, anti-phase coordination pattern. Infants got faster with prior cruising experience because they mastered a specific coordination pattern-a trot-like gait—not because they increased the speed and amplitude of individual limb movements regardless of coordination. However, the anti-phase coordination pattern was rare 
in Experiment 2 when the environment was variable (varying distance between two handrails). Both novice and experienced cruisers displayed a variety of coordination patterns to cross from one handrail to the other, and experience facilitated solutions by teaching infants to use the appropriate pattern at the appropriate time. Moreover, experienced cruisers used the same coordination pattern for the same distance and different patterns for different distances, whereas novice cruisers switched coordination patterns from trial to trial while crossing the same distance.

The diversity of coordination patterns in novice cruisers supports the emergentist viewthat development begins by generating multiple solutions regardless of the environment; and with experience, infants learn to select coordination patterns that are specific to the relations between body and environment. If the nativist view were correct that infants begin with a prescribed solution and experience elaborates on that solution, novice cruisers should have used the same coordination pattern over and over. But they did not. Instead, novice cruisers tried different strategies even for the same distance between handrails.

Our findings expand on previous work that points to everyday experience as fundamental for facilitating infants' ability to solve locomotor problems (for reviews, see $[4,6]$ ). As in the current study, previous work showed that novice walkers take large and small, fast and slow steps indiscriminately while walking down slopes; they do not tailor gait patterns to the degree of slant, so their momentum increases uncontrollably on steep slopes. Over weeks of everyday walking experience, infants learn to precisely match their step length and speed to variations in slant in real time, and they plan their gait modifications prospectively before stepping over the brink [33]. Use of real-time analyses and machine-learning algorithms in the current study revealed how infants acquire this ability. They start by generating a variety of solutions and over weeks of experience, they learn to assemble the appropriate coordination pattern on the fly. 


\section{Specificity of Experience}

A robust finding in both experiments was the specificity of infants' prior experience. Only cruising, not crawling experience, predicted infants' performance, despite the fact that crawling involves an anti-phase coordination pattern among the four limbs $[10,14,19]$ and typically precedes cruising [18]. Thus, experience with an earlier developing skill does not transfer automatically to a later developing skill. Learning is specific to the different problems of crawling and cruising. The dissociation between postures supports a growing body of research showing that infants' success is predicted by their experience maintaining balance in each posture, not by their age or duration of general locomotion experience [for reviews, see 4, 5, 6].

What constitutes infants' prior cruising experience? It is unlikely that everyday experience involves long stretches of anti-phase interlimb coordination because-unlike the $302-\mathrm{cm}$ long handrail in the lab-the everyday world is not populated with long, continuous surfaces at infants' chest height. Moreover, everyday experience is unlikely to entail practice cruising over the specific body-distance ratios we tested in the lab because the distance between furniture is variable from one home to the next and infants' body size changes from week to week [34]. Thus, it is unlikely that infants learned particular solutions from everyday life and then applied them in the lab. Rather, we suggest that everyday cruising experience coping with changes in the body and environment promotes "learning to learn," where infants learn to assemble the appropriate strategy for new problems in real time. More generally, infant data and robot simulations based on infant data indicate that varied motor experience promotes adaptive, real-time solutions for sitting, crawling, cruising, and walking [17, 33, 35-37]. Future work should investigate which aspects of variable experience promote learning to learn.

\section{Conclusion}

How do infants acquire the flexibility to solve locomotor problems in real time like cruising over varying distances between supports? As predicted by an emergentist view, 
inexperienced infants generate multiple solutions, some better, some worse, and do the best they can. Over weeks of everyday experience with the relevant motor skill-all the while presumably coping with variations in the body and environment-infants learn to assemble solutions in real time as they encounter new problems.

\section{Author Contributions}

Both authors contributed to the study concept, design, and interpretation. KEA collected the data. OO developed and performed the analyses. Both authors wrote the manuscript and approved the final version of the manuscript for submission.

\section{Acknowledgments}

This work was supported by NSF/SBE-BSF grant \#1627993, NICHD grant \#R01HD086034, and DARPA grant \#N66001-19-2-4035 to Karen Adolph. We thank Minxin Cheng for her beautiful line drawings; Melody Xu for help with video coding; and Mark Blumberg and Anastasia Shuster for their useful suggestions.

Declaration of Interests

The authors declare no competing interests.

\section{STAR Methods}

\section{Lead Contact and Materials Availability}

Requests for further information and data resources should be directed to and will be fulfilled by the Lead Contact, Karen E. Adolph (kea1@nyu.edu). This study did not generate new reagents.

\section{Data and Code Availability}

All third-person videos of infants' behaviors and pose estimations and the video coding manual are openly shared with authorized investigators in Databrary (https://nyu.databrary.org/volume/1116). The analysis codes are shared publicly on Github (https://anonymous.4open.science/r/1116/). 


\section{Experimental Model and Subject Details}

\section{Participants}

Parents of all participating infants gave written informed consent prior to participation. All experiments were approved by the ethics board of New York University. Families were recruited from maternity wards of local hospitals in the NYC area and received small souvenirs for participation.

Experiment 1. Twenty-four 11-month-old infants (10.84-11.34 months; 10 boys) participated. All infants could cruise and crawl. Based on parent report, infants had $M=49.7$ days of cruising experience (range $=13-103$ days) and $M=72.3$ days of crawling experience (range = 29-119 days), dating from the first day infants cruised 1 meter continuously along a couch or coffee table and crawled 3 meters on hands and knees continuously across the floor $[17,23]$; Figure S1A. An experimenter measured infants' recumbent height, nude weight, leg length (from hip to ankle), and wingspan (outstretched arms from fingertip to fingertip) as shown in supplemental Table S1. Data from 7 additional infants were not analyzed due to infants fussing $(n=6)$ or video equipment failure $(n=1)$.

Experiment 2. Twenty-two 11-month-old infants ( \pm 2 weeks; $10.52-11.63$ months, 12 boys) participated. Parents reported that infants had $M=58.68$ days of cruising experience (range $=6-122$ days) and $M=88.78$ days of crawling experience (range $=14-170$ days); see Figure S1B.

\section{Method Details}

\section{Procedure and Apparatus}

Experiment 1. Infants cruised sideways (facing handrail and holding it with both hands) over a 302-cm long $\times 14-\mathrm{cm}$ wide wooden handrail on a raised platform covered with plush carpet. The height of the handrail $(41 \mathrm{~cm})$ was approximately at infants' chest level, $55 \%$ of their average standing height. Each infant contributed four cruising trials to analyses. Trials began 
with infants standing at one end of the platform and ended after infants cruised to the other end of the platform. An experimenter followed alongside infants to ensure their safety. Infants' movements were recorded with a panning camera (30 fps) perpendicular to the handrail (see Figure $1 \mathrm{~A}$ and https://nyu.databrary.org/volume/1116). We aimed for 4 trials per infant, but due to occasional fussiness, we obtained $M=3.54$ trials per infant.

Experiment 2. As in Experiment 1, infants cruised sideways over a wooden handrail on the raised platform. However, as shown in Figure 4A, the handrail was composed of two $105-\mathrm{cm}$ long segments that could be separated to create 0 - to $68-\mathrm{cm}$ wide distances between handrails $[17,23]$. Cruising over the gap between handrails required infants to position their arms and legs appropriately on the edge of the first handrail so they could grasp the edge of the second handrail without losing balance. Trials began with infants holding the first handrail about $10 \mathrm{~cm}$ away from the edge (Figure 4A). The distance between handrails varied according to an adaptive psychophysical procedure that provided more and less challenging distances based on infants' success at cruising on the last trial [17].

\section{Data Coding}

Experiment 1. Because infants sometimes began cruising slightly beyond the start of the handrail and/or stopped cruising momentarily as they moved along the handrail, we analyzed infants' movements for a $69-\mathrm{cm}$ segment of the handrail defined by permanent posts on the platform. A primary coder used Datavyu software (www.datavyu.org) to identify the first video frame when infants' leading leg moved past the first post and the first video frame when their trailing leg moved past the second post. The coder also scored the number of times infants lifted and set down each foot and each hand. A second coder independently rescored all the trials. Inter-observer agreement ranged from 89-96\%, Cohen's $\kappa s>.86, p s<.00$.

Experiment 2. Coders used Datavyu to identify trials when infants cruised successfully over the handrail distance. Trials were considered successful if infants cruised safely from one 
handrail to another. Trials were unsuccessful if infants attempted to cruise but fell into the gap between the handrails, avoided crossing for the 30 -s trial, or crawled from one handrail to the other. Only successful trials were analyzed further. Next, coders scored the onset and offset of each trial. Trial onset was the first video frame when infants' arm or leg moved past the edge of the starting handrail. Trial offset was the first video frame when both arms were on the ending handrail. A second coder scored $25 \%$ of the trials for each infant. Coders agreed on success for $98 \%$ of trials, Cohen's $\kappa>.78, p<.00$; the correlation coefficient for cruising time was $r=.95, p$ $<.00$.

\section{Movement Time Series}

Experiment 1. We used AutoViDev—a video-analysis tool that uses computer vision to support video-based developmental research [24]—to automatically detect infants' movements and to formalize cruising as a time series (Figure 2 and https://nyu.databrary.org/volume/1116). The software applied a convolutional pose machine algorithm [38] based on real-time 2D pose estimation using part affinity fields, OpenPose [31, 32]. Computer-vision detection of infants' limb positions was successful in all the trials, although the algorithm occasionally lost a frame or two.

Then, as shown in Figure 2C, for each video frame, we calculated the Euclidean distance of each pair of limbs (the two hands and the two feet), where $x, y$ signifies the location of the key points in a video frame:

(a) $\quad d_{\text {arms }}=\left(x_{\text {right_hand }}-x_{\text {left_hand }}\right)^{2}+\left(y_{\text {right_hand }}-y_{\text {left_hand }}\right)^{2}$

(b) $\quad d_{\text {legs }}=\left(x_{\text {right_foot }}-x_{\text {left_foot }}\right)^{2}+\left(y_{\text {right_foot }}-y_{\text {left_foot }}\right)^{2}$

Change in these distances across video frames characterizes the moment-to-moment coordination in cruising. For example, the distance between the hands increases as infants stretch out their arms and it decreases when they bring their arms together. Similarly, the distance between the feet increases when infants take a step and decreases when they take 
another step by closing their legs. Therefore, we created a movement time series (MTS) for each trial based on changes in the hands and feet distances, where $n$ is the number of frames in trial $t$ :

(c) $\quad$ arm_MTS $S_{t}=\left[\left(d_{\text {arms }}\right)_{1},\left(d_{\text {arms }}\right)_{2} \ldots \ldots\left(d_{\text {arms }}\right)_{n}\right]$

(d) $\quad$ leg_MTS $_{t}=\left[\left(d_{\text {legs }}\right)_{1},\left(d_{\text {legs }}\right)_{2} \ldots \ldots\left(d_{\text {legs }}\right)_{n}\right]$

To reduce signal noise, we smoothed the change in distance by: (a) calculating a z-score for each trial; (b) down-sampling every two frames; and (c) applying a one-dimensional median filter in $2^{\text {nd }}$ order for both arms and legs. Importantly, each MTS reflects temporal distance changes between infant's limbs, and not absolute values of those distances. Therefore, differences between MTSs are not affected by differences in camera location or experimenterinduced movements of the camera across trials and participants. Figure 2D shows examples of MTS for an experienced cruiser (top) and novice cruiser (bottom).

Experiment 2. As in Experiment 1, computer vision detection of infants' movements was successful in all trials. For Experiment 2, we formalized cruising trials as a multivariate MTS [39] that combined changes in arms and legs, where $n$ is the number of frames in trial $t$ :

(e) $\quad \operatorname{MTS} S_{t}=\left[\left(d_{\text {arms }}, d_{\text {legs }}\right)_{1},\left(d_{\text {arms }}, d_{\text {legs }}\right)_{2} \ldots \ldots\left(d_{\text {arms }}, d_{\text {legs }}\right)_{n}\right]$ We reduced signal noise similarly to Experiment 1.

\section{Dynamic Time Warping}

In Experiment 2, infants cruised across different handrail distances. Therefore, each multivariate MTS differed in length (Figure 5A). We used dynamic time warping (DTW) to calculate the similarity between trials across infants and distances. DTW is a non-linear sequence alignment algorithm [40] widely used in machine-learning processes for speech recognition, bioinformatics, and handwriting identification [41, 42]. DTW of multivariate time series provides a robust similarity measure that indicates the similarity in coordination patterns between each pair of trials $[43,44]$. Similarity was defined as 1 over the cumulative DTW 
measure normalized by the length of the DTW path. Figure S6 shows the DTW process for calculating similarity between trials with similar (left) and not similar (right) coordination patterns.

\section{Density Peak Clustering}

To identify real-time strategies in Experiment 2, we used DTW to calculate similarity between each pair of trials. Then, we constructed a movement similarity matrix —an m-by-m matrix where the ( $\mathrm{i}$-th , $\mathrm{j}$-th) cell of the matrix contains the similarity index between trial $\mathrm{i}$ and trial $\mathrm{j}$, and $\mathrm{m}$ is the total number of successful trials across infants and handrail distances. Thus, each cell in the similarity matrix indicates the degree to which each pair of trials is similar. We then clustered trials across infants and handrail distances based on their MTSs. As shown in previous work $[45,46]$, this method is agnostic about the number of clusters, and the reason for clustering must be interpreted posthoc based on the movement patterns.

The algorithm identifies several MTS as "strategy prototypes" and then classifies the rest of the trials according to the strategy prototype to which they were closest in the similarity matrix. A prototype trial was characterized by (1) a high number of MTS that were relatively similar (the "density coefficient"); and (2) a low similarity to MTS with higher density coefficients ("distance coefficient").

The density coefficient reflects the number of MTS whose similarity to MTSi in the similarity matrix is higher than a pre-defined cutoff similarity based on the potential entropy of the data field [47]. Formally, the density coefficient $\rho_{i}$ is estimated by the following formula, where the function $\chi$ is defined such that $\chi=1$ if $s_{i, j}-s_{c}>0$ and $\chi=0$ otherwise; $s_{i, j}$ represents the similarity between MTSi and MTSj and $s_{c}$ represents the cutoff similarity:

$$
\text { (f) } \quad \rho_{i}=\sum_{j=1}^{m} \chi\left(s_{i, j}-s_{c}\right)
$$

The distance coefficient $\delta_{i}$ reflects the maximal similarity between each MTS and the next MTS with a higher density coefficient:

(g) $\quad \delta_{i}=\max _{j: \rho_{j}>\rho_{i}}\left(s_{i j}\right)$ 
The MTS with the highest density was assigned the minimum value. The distance coefficient and the density coefficient were multiplied to create a y score for each trial. We chose prototypes as the outliers from the $y$ distribution of all trials (outliers are values for which $\mathrm{y}$ was at least three standard deviations above the mean). The number of outliers determined the number of clusters. All other trials were assigned to the prototype trial to which they were most similar.

\section{Quantification and Statistical Analysis}

\section{Real-Time Movement Variability}

In Experiment 1, we assessed variability in step durations based on the phase changes in the arm and leg MTS. For each trial, we calculated variability in phase change in the MTS for each pair of limbs independently. First, we extracted phase using a Hilbert transform, a linear operator that splits the time series into its phase and power. Then, we calculated the difference in phase angle between each two time points. If the difference in phase angle was not equal to 0 , we considered the time point as a step onset. We calculated the duration between each pair of step onsets. Variability was then defined as the standard deviation of these durations. For each infant, we averaged the standard deviation across trials.

\section{Real-Time Measures of Coordination}

In Experiment 1, we assessed infants' real-time coordination patterns using three measures that compare the arm and leg MTS: (1) Maximum cross-correlation coefficient, (2) Pearson correlation, and (3) phase-locking value. Cross correlation between any two time series measures their similarity as a function of time shift. The cross-correlation function is peaked at times when the time series are most aligned. The maximum cross-correlation coefficient reflects the maximum similarity between the two time series across all time lags. Therefore, we selected the maximum cross-correlation coefficient as our measure of similarity between arm and leg movements. 
The linear correlation between the two MTS was represented by a Pearson correlation value ranging from -1 (negative alignment between arm and leg movements) to 0 (no alignment) to 1 (perfect alignment). The instantaneous phase locking value (PLV) represented the consistency between arm and leg MTS. PLV is widely used in neuroscience to evaluate the phase difference between two brain signals [48]. Phases of two signals are "locked" (i.e., high PLV) if their difference is constant over time. Thus, this measure reflects infants' stability in coordinating arms and legs over the trial. This technique allowed us to compute stability without arbitrarily defining a window size as done in moving correlations [49].

\section{Switching Index}

In Experiment 2, we examined whether infants switched real-time strategies for each handrail distance. Because adjacent distances were similar (e.g., 28 and $30 \mathrm{~cm}$ ), we systematically calculated a switching index for different sliding window sizes. We tested 2-cm, 4$\mathrm{cm}, 8-\mathrm{cm}, 12-\mathrm{cm}$, and $16-\mathrm{cm}$ windows. For each infant, we computed the number of different strategies in each window size, sliding along in 2-cm increments from 10 to $68 \mathrm{~cm}$. Thus, for the 2-cm window size, we computed the number of different strategies used when the distance was $10 \mathrm{~cm}$, then $12 \mathrm{~cm}$, and so on. For the 4-cm window, we computed the number of different strategies when distance ranged from 10-14 cm, then from $12-16 \mathrm{~cm}$, and so on. For the $16-\mathrm{cm}$ window size, we computed the number of different strategies when distance ranged from 10-26 $\mathrm{cm}, 12-28 \mathrm{~cm}$, and so on. 


\section{References}

1. Winter, D.A. (1995). A.B.C. of balance during standing and walking, (Waterloo: Waterloo Biomechanics).

2. Bernstein, N.A. (1996). On dexterity and its development. In Dexterity and its development, M.L. Latash and M.T. Turvey, eds. (Mahwah, NJ: Erlbaum), pp. 3-244.

3. Patla, A.E. (1991). Visual control of human locomotion. In Adaptability of human gait, A.E. Patla, ed. (North-Holland: Elsevier Science Publishers), pp. 55-97.

4. Adolph, K.E., and Hoch, J.E. (2019). Motor development: Embodied, embedded, enculturated, and enabling. Annual Review of Psychology 70, 141-164.

5. Adolph, K.E., Hoch, J.E., and Cole, W.G. (2018). Development (of walking): 15 suggestions. Trends in Cognitive Sciences 22, 699-711.

6. Adolph, K.E., and Robinson, S.R. (2015). Motor development. In Handbook of child psychology and developmental science, Volume 2 Cognitive Processes, 7th Edition, L. Liben and U. Muller, eds. (New York: Wiley), pp. 113-157.

7. Kretch, K.S., and Adolph, K.E. (2017). The organization of exploratory behaviors in infant locomotor planning. Developmental Science 20, e12421.

8. Spelke, E.S., and Newport, E.L. (1998). Nativism, empiricism, and the development of knowledge. In Handbook of child psychology: Vol. 1. Theoretical models of human development, 5th Edition, W. Damon, ed. (New York: John Wiley \& Sons, Inc), pp. 275340.

9. Dominici, N., Ivanenko, Y.P., Cappellini, G., d'Avella, A., Mondi, V., Cicchese, M., Fabiano, A., Silei, T., Di Paolo, A., Giannini, C., et al. (2011). Locomotor primitives in newborn babies and their development. Science 334, 997-999.

10. Patrick, S.K., Noah, J.A., and Yang, J.F. (2009). Interlimb coordination in human crawling reveals similarities in development and neural control with quadrupeds. Journal of Neurophysiology 101, 603-613.

11. Forma, V., Anderson, D.I., Provasi, J., Soyez, E., Martial, M., Huet, V., Granjon, L., Goffinet, F., and Barbu-Roth, M. (2019). What does prone skateboarding in the newborn tell us about the ontogeny of human locomotion? Child Development 90, 1286-1302.

12. Forssberg, H. (1985). Ontogeny of human locomotor control. I. Infant stepping, supported locomotion, and transition to independent locomotion. Experimental Brain Research 57, 480-493.

13. Thelen, E., and Ulrich, B.D. (1991). Hidden skills: A dynamic systems analysis of treadmill stepping during the first year. Monographs of the Society for Research in Child Development 56, 1-103.

14. Freedland, R.L., and Bertenthal, B.I. (1994). Developmental changes in interlimb coordination: Transition to hands-and-knees crawling. Psychological Science 5, 26-32.

15. Thelen, E. (1991). Timing in motor development as emergent process and product. In Advances in psychology, Volume 81, J. Fagard and P.H. Wolff, eds. (Amsterdam: Elsevier), pp. 201-211.

16. Clark, J.E., and Phillips, S.J. (1993). A longitudinal study of intralimb coordination in the first year of independent walking: A dynamical systems analysis. Child Development 64, 1143-1157.

17. Adolph, K.E., Berger, S.E., and Leo, A.J. (2011). Developmental continuity? Crawling, cruising, and walking. Developmental Science 14, 306-318.

18. Martorell, R., Onis, M., Martines, J., Black, M., Onyango, A., and Dewey, K.G. (2006). WHO motor development study: Windows of achievement for six gross motor development milestones. Acta Paediatrica 95 (S450), 86-95. 
19. Cole, W.G., Vereijken, B., Young, J.W., Robinson, S.R., and Adolph, K.E. (2019). Use it or lose it? Effects of age, experience, and disuse on quadrupedal gait. Developmental Psychobiology 61, 29-42.

20. Adolph, K.E., Vereijken, B., and Denny, M.A. (1998). Learning to crawl. Child Development 69, 1299-1312.

21. Vereijken, B., and Adolph, K.E. (1999). Transitions in the development of locomotion. In Non-linear analyses of developmental processes, G.J.P. Savelsbergh, H.L.J. van der Maas and P.C.L. van Geert, eds. (Amsterdam: Elsevier), pp. 137-149.

22. Haehl, V., Vardaxis, V., and Ulrich, B.D. (2000). Learning to cruise: Bernstein's theory applied to skill acquisition during infancy. Human Movement Science 19, 685-715.

23. Berger, S.E., Chan, G., and Adolph, K.E. (2014). What cruising infants understand about support for locomotion. Infancy 19, 117-137.

24. Ossmy, O., Gilmore, R.O., and Adolph, K.E. (2020). AutoViDev: A computer-vision framework to enhance and accelerate research in human development. In Advances in computer vision: CVC 2019. Advances in Intelligent Systems and Computing, Volume 944, K. Arai and S. Kapoor, eds. (Cham, Switzerland: Springer), pp. 147-156.

25. Vogt, N. (2018). Machine learning in neuroscience. Nature Methods 15, 33.

26. Berger, S.E., Adolph, K.E., and Lobo, S.A. (2005). Out of the toolbox: Toddlers differentiate wobbly and wooden handrails. Child Development 76, 1294-1307.

27. Winter, D.A. (2009). Biomechanics and motor control of human movement, (Hoboken, NJ: John Wiley \& Sons).

28. Edelman, S. (1998). Representation is representation of similarities. Behavioral and Brain Sciences 21, 449-467.

29. Shepard, R.N. (1980). Multidimensional scaling, tree-fitting, and clustering. Science 210, 390-398.

30. Kriegeskorte, N., Mur, M., and Bandettini, P.A. (2008). Representational similarity analysis-connecting the branches of systems neuroscience. Frontiers in Systems Neuroscience 2, 4.

31. Wei, W.E., Ramakrishna, V., Kanade, T., and Sheikh, T. (2016). Convolutional pose machines. In Proceedings of the IEEE conference on Computer Vision and Pattern Recognition. pp. 4724-4732.

32. Simon, T., Joo, H., Matthews, I., and Sheikh, Y. (2017). Hand keypoint detection in single images using multiview bootstrapping. In Proceedings of the IEEE conference on Computer Vision and Pattern Recognition. pp. 1145-1153.

33. Gill, S.V., Adolph, K.E., and Vereijken, B. (2009). Change in action: How infants learn to walk down slopes. Developmental Science 12, 888-902.

34. Lampl, M., Veldhuis, J.D., and Johnson, M.L. (1992). Saltation and stasis: A model of human growth. Science 258, 801-803.

35. Rachwani, J., Soska, K.C., and Adolph, K.E. (2017). Behavioral flexibility in learning to sit. Developmental Psychobiology 59, 937-948.

36. Adolph, K.E. (1997). Learning in the development of infant locomotion. Monographs of the Society for Research in Child Development 62, 1-140.

37. Ossmy, O., Hoch, J.E., MacAlpine, P., Hasan, S., Stone, P., and Adolph, K.E. (2018). Variety wins: Soccer-playing robots and infant walking. Frontiers in Neurorobotics 12, 19.

38. Ramakrishna, V., Munoz, D.P., Hebert, M., Bagnell, J.A., and Sheikh, Y. (2014). Pose machines: Articulated pose estimation via inference machines. In European Conference on Computer Vision. (Springer), pp. 33-47.

39. Reinsel, G.C. (2003). Elements of multivariate time series analysis, (Springer Science \& Business Media). 
40. Berndt, D.J., and Clifford, J. (1994). Using dynamic time warping to find patterns in time series. KDD workshop 10, 359-370.

41. Myers, C.S., and Rabiner, L.R. (1981). A comparative study of several dynamic timewarping algorithms for connected-word recognition. Bell System Technical Journal 60, 1389-1409.

42. Muda, L., Begam, M., and Elamvazuthi, I. (2010). Voice recognition algorithms using mel frequency cepstral coefficient (MFCC) and dynamic time warping (DTW) techniques. arXiv preprint arXiv:1003.4083.

43. Keogh, E.J., and Pazzani, M.J. (2000). Scaling up dynamic time warping for datamining applications. Proceedings of the sixth ACM SIGKDD international conference on Knowledge discovery and data mining, 285-289.

44. Bankó, Z., and Abonyi, J. (2012). Correlation based dynamic time warping of multivariate time series. Expert Systems with Applications 39, 12814-12823.

45. Karasik, L.B., Tamis-LeMonda, C.S., Ossmy, O., and Adolph, K.E. (2018). The ties that bind: Cradling in Tajikistan. PLoS ONE 13, e0204428.

46. Rodriguez, A., and Laio, A. (2014). Clustering by fast search and find of density peaks. Science 34, 1492-1496.

47. Wang, S., Wang, D., and Li, Y. (2015). Comment on "Clustering by fast search and find of density peaks". arXiv preprint arXiv:1501.04267.

48. Lachaux, J.P., Rodriguez, E., Martinerie, J., and Varela, F.J. (1999). Measuring phase synchrony in brain signals. Human Brain Mapping 8, 194-208.

49. Boker, S.M., Rotondo, J.L., Xu, M., and King, K. (2002). Windowed cross-correlation and peak picking for the analysis of variability in the association between behavioral time series. Psychological Methods 7, 338. 
Figures
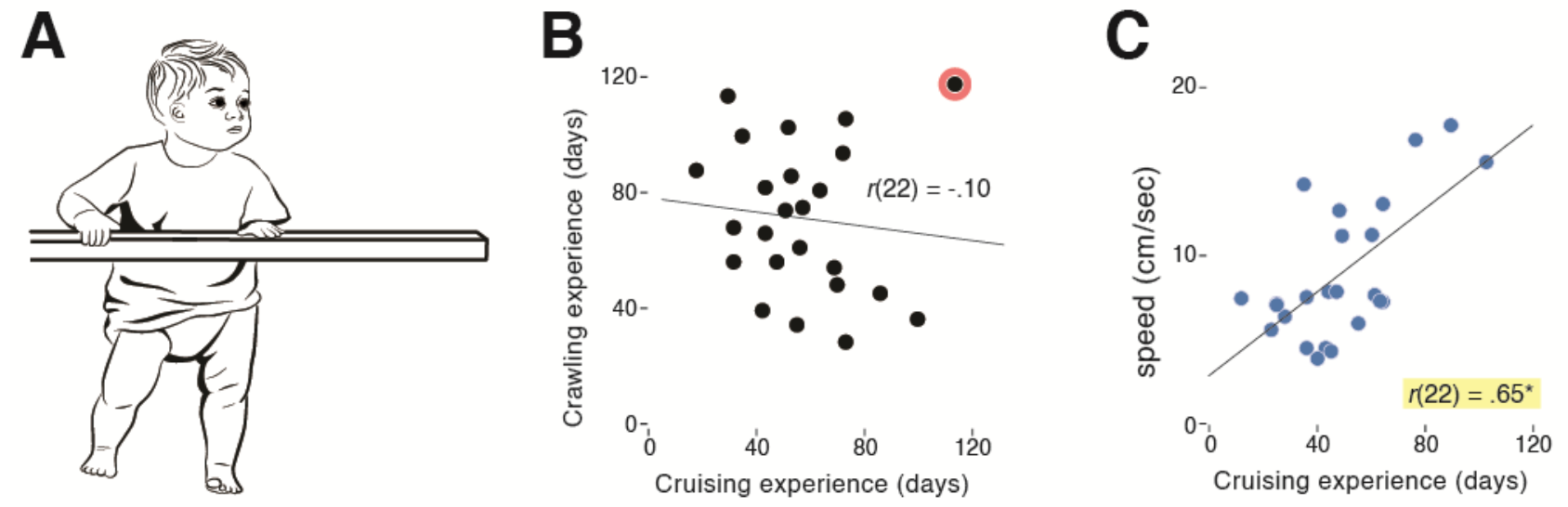

Figure 1. Experiment 1 design and results. (A) Apparatus used to test infants cruising along a continuous handrail they held for support. (B) Crawling and cruising experience were not correlated. After excluding the outlier (marked in red), the correlation improved, but was not significant, $r(21)=-.39, p=.06$. (C) Cruising experience predicted infants' speed. 

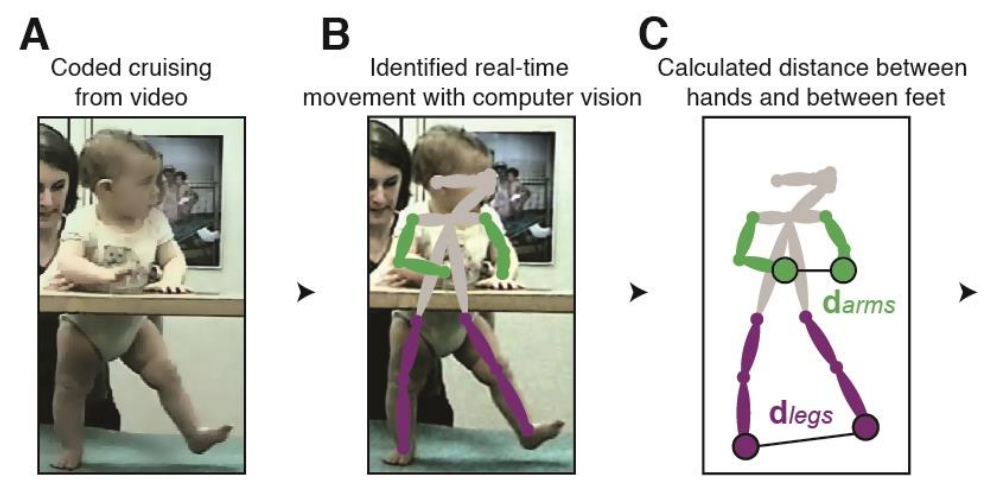

D

E

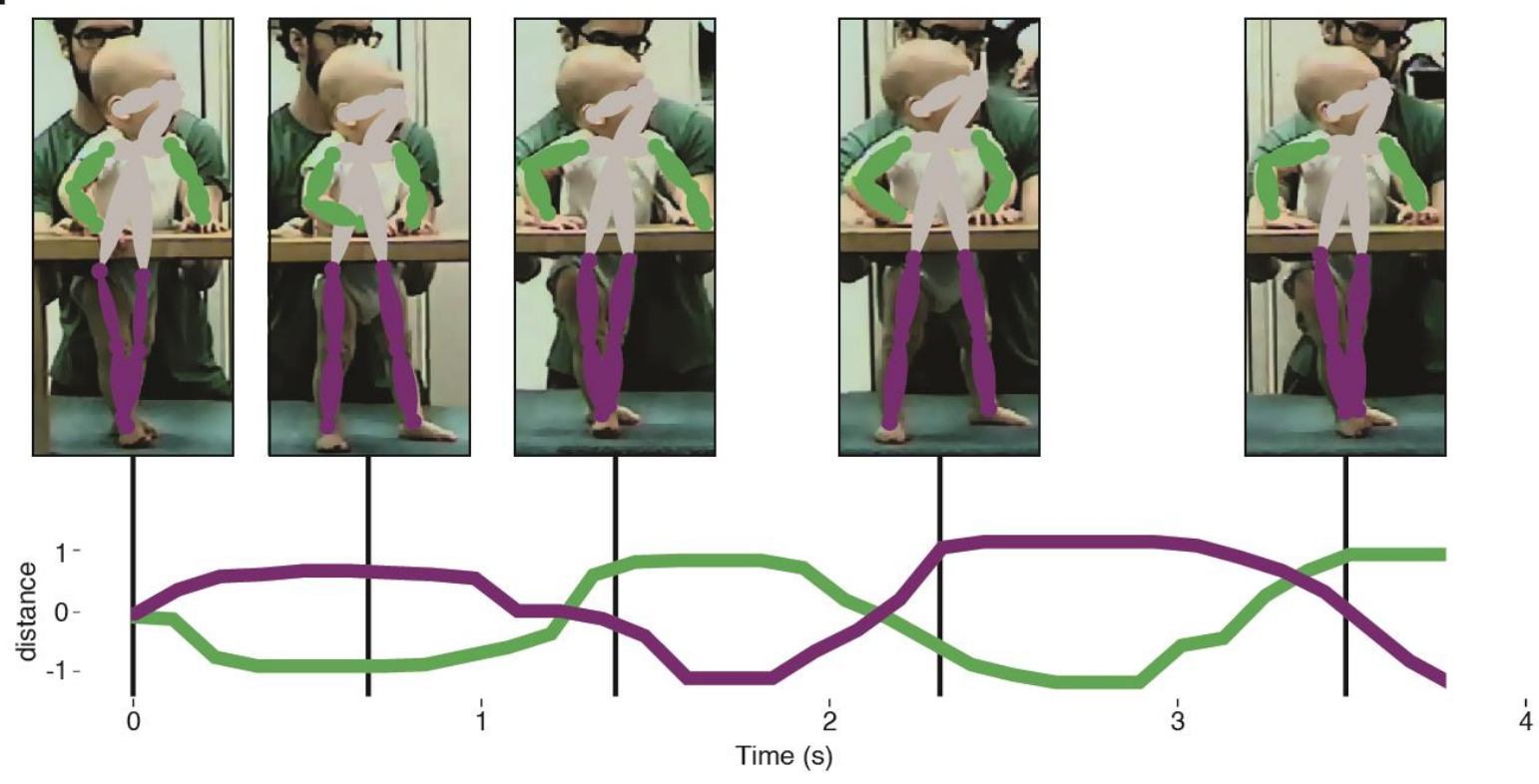

Figure 2. Experiment 1: Movement time series for arms and legs. (A) Human coders manually scored videos for trials in which infants successfully crossed the distance between handrails. (B) A computer vision algorithm tracked body movement frame-by-frame (colored skeleton presents an example of data from one video frame). (C) For each video frame, we calculated the distance between the limbs (darms for the distance between the hands and dlegs for the distance between the feet), to extract the real-time coordination pattern for cruising. (D) Movement time series representing a trial by the most experienced cruiser (103 days; top) and the least experienced cruiser ( 13 days; bottom). More experience led to faster performance and more stable arm-leg coordination. (E) Example of an anti-phase strategy in $4 \mathrm{~s}$ of cruising, in which infants open their legs (by stepping the leading leg sideways) and close their arms (by bringing the trailing arm next to the leading arm) at the same time, and then close their legs (by bringing the trailing leg next to the leading leg) and open their arms (by moving the leading arm sideways) at the same time in an oscillatory manner. 


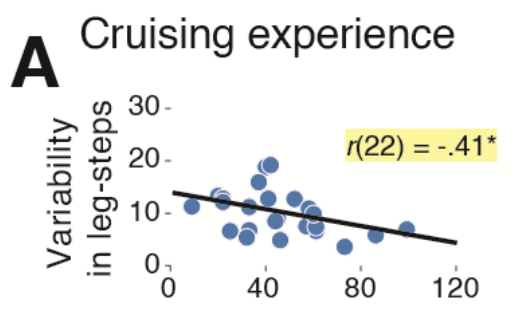

Crawling experience

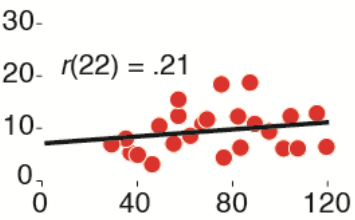

B
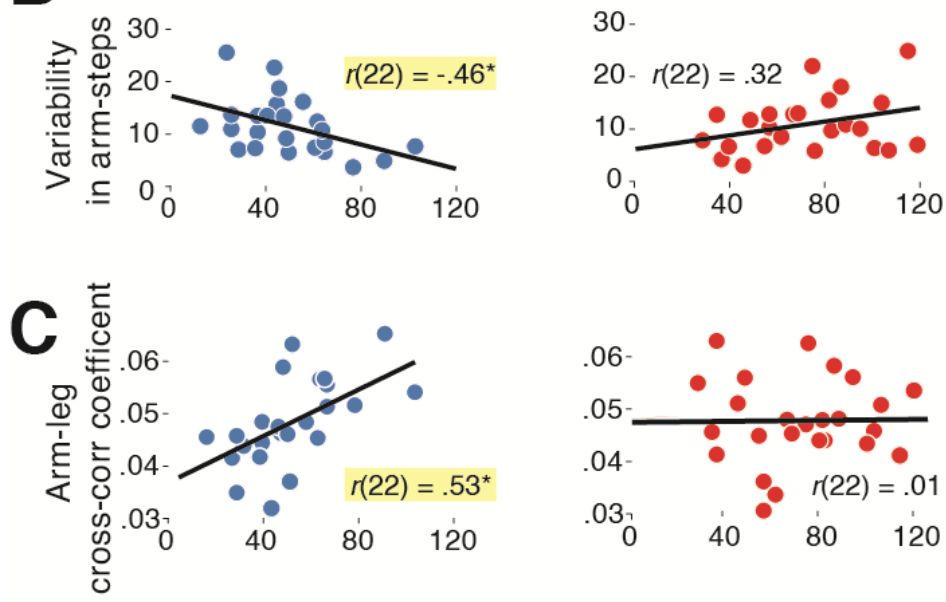

D
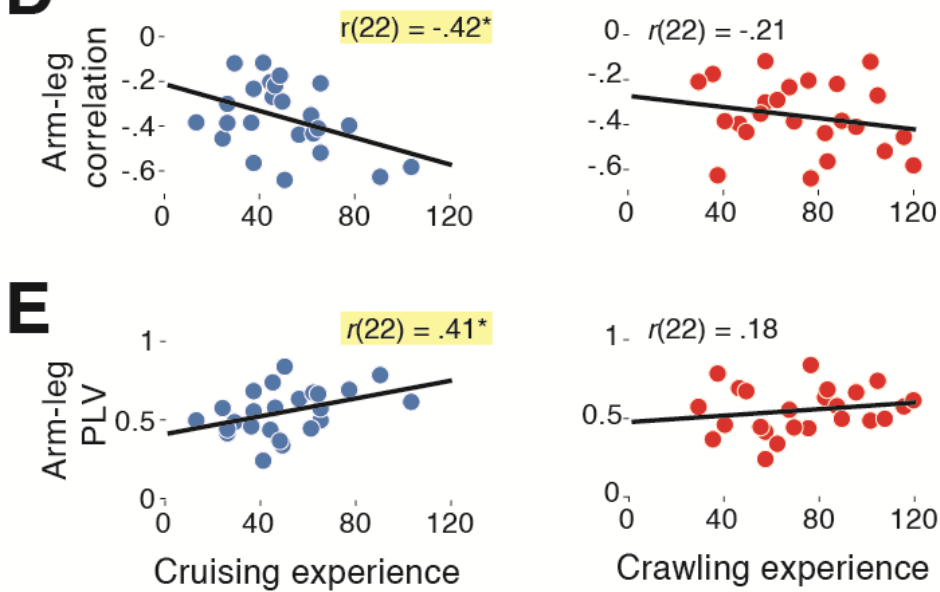

(days)

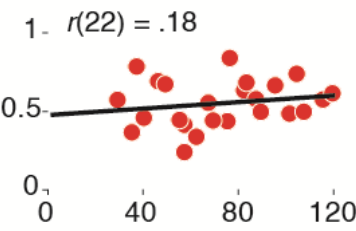

Crawling experience (days)

Figure 3. Experiment 1: Scatterplots showing correlations between cruising experience and crawling experience, and five measures of interlimb coordination. Significant correlations are highlighted in yellow ( $p s<.05)$. (A) Variability in leg movements ( $S D$ of step duration) decreased with cruising experience, but not with crawling experience. (B) Variability in arm movements, like leg movements, decreased with cruising experience, but not with crawling experience. (C) Similarity (maximal cross-correlation coefficient) between arm and leg movement time series increased with cruising experience, but not with crawling experience. (D) The negative correlations between arm and leg movements (indicating a precise anti-phase coordination pattern) increased with cruising experience but did not vary with crawling experience. (E) The phase-locking value (see STAR Methods) was positively correlated with cruising experience, but not with crawling experience. 
A

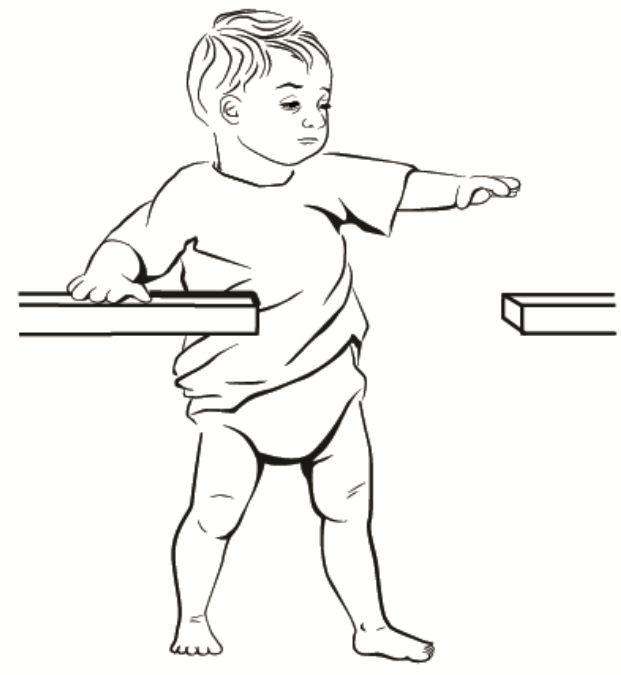

B

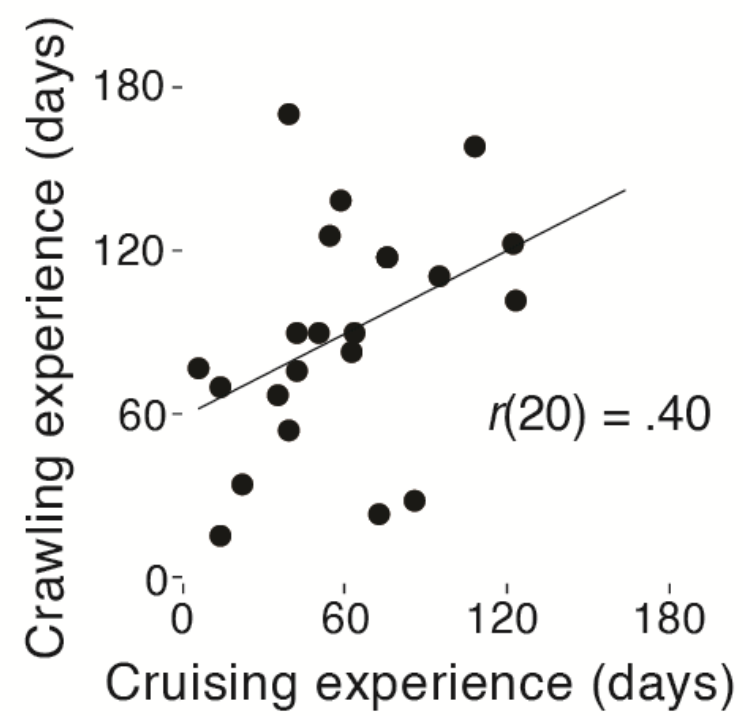

Figure 4. Experiment 2 design. (A) Apparatus used to test infants cruising between two handrails they held for support. (B) Cruising and crawling experience were not correlated. 
A

Similarity based on movement time series

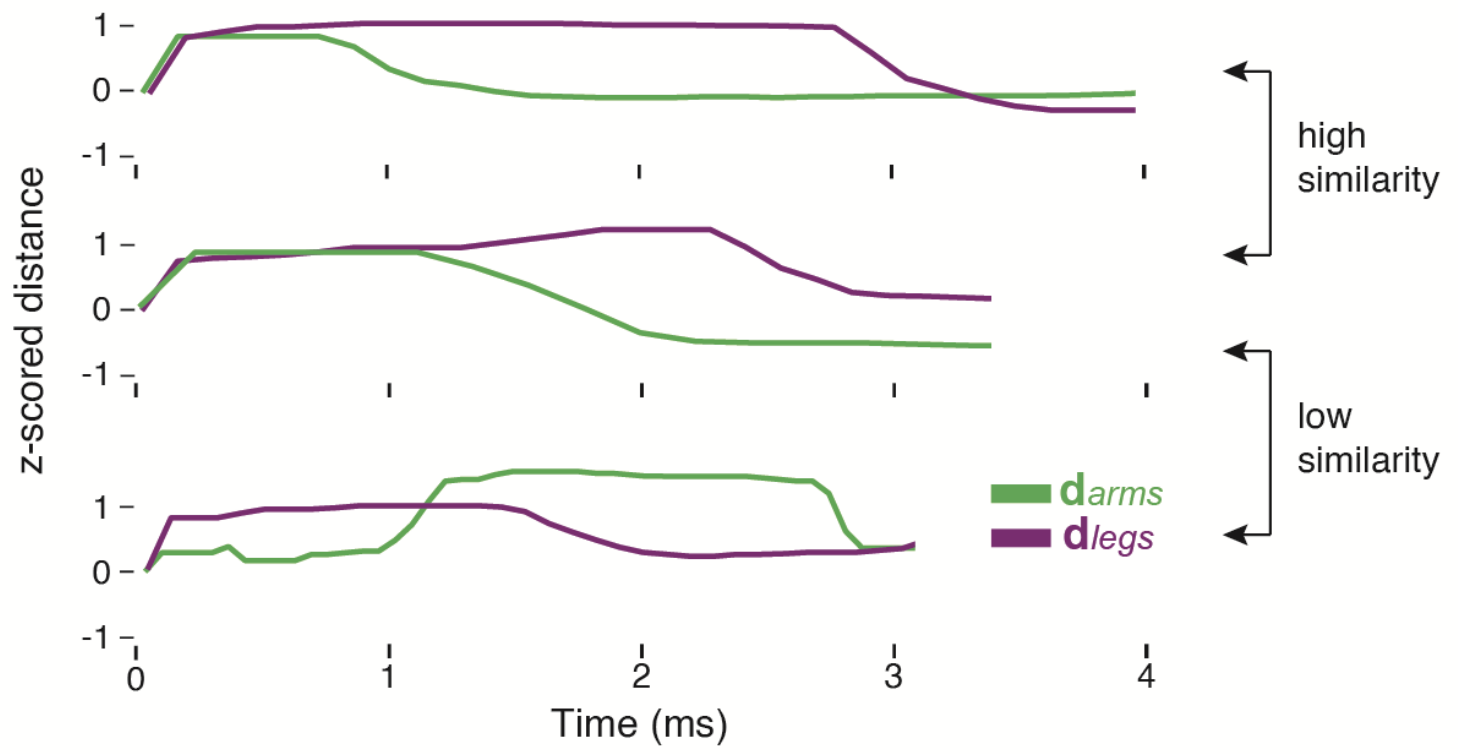

B

Two dimensional similarity space

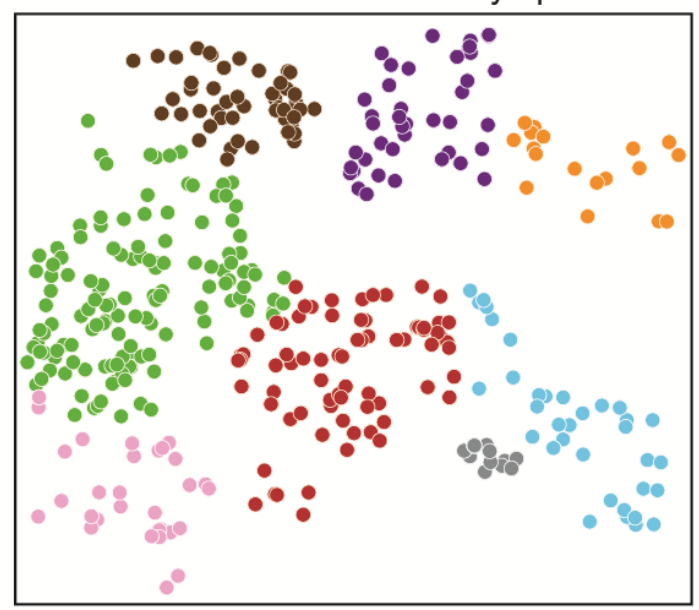

Similarity matrix ordered by clusters

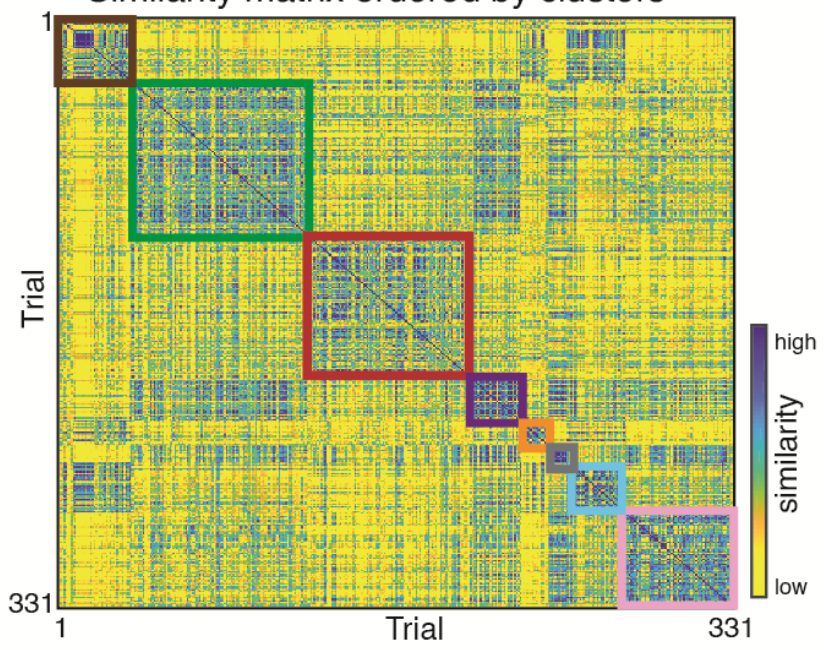

Figure 5. Experiment 2: Movement similarity matrices. (A) Movement time series representing trials in three different infants. Two trials (top and middle) had high similarity (both graphs show a coordination strategy in which infants increased the distance between legs and arms, then decreased leg distance, and then decreased arm distance), and two trials (middle and bottom) had low similarity (bottom graph shows a different strategy in which the infant increased the distance between arms, then increased leg distance, then decreased arm distance, then decreased leg distance). (B) Clustering based on the similarity among trials. Left panel shows all trials in a two-dimensional space. Each trial is represented by a symbol, and the distance between every two points is the similarity between them (e.g., points that are closer together represent more similar trials). Trials are colored according to clusters. Right panel shows the movement similarity matrix ordered according to clusters. The blue squares along the diagonal show high similarity among trials within clusters (square borders match cluster colors in left panel). 

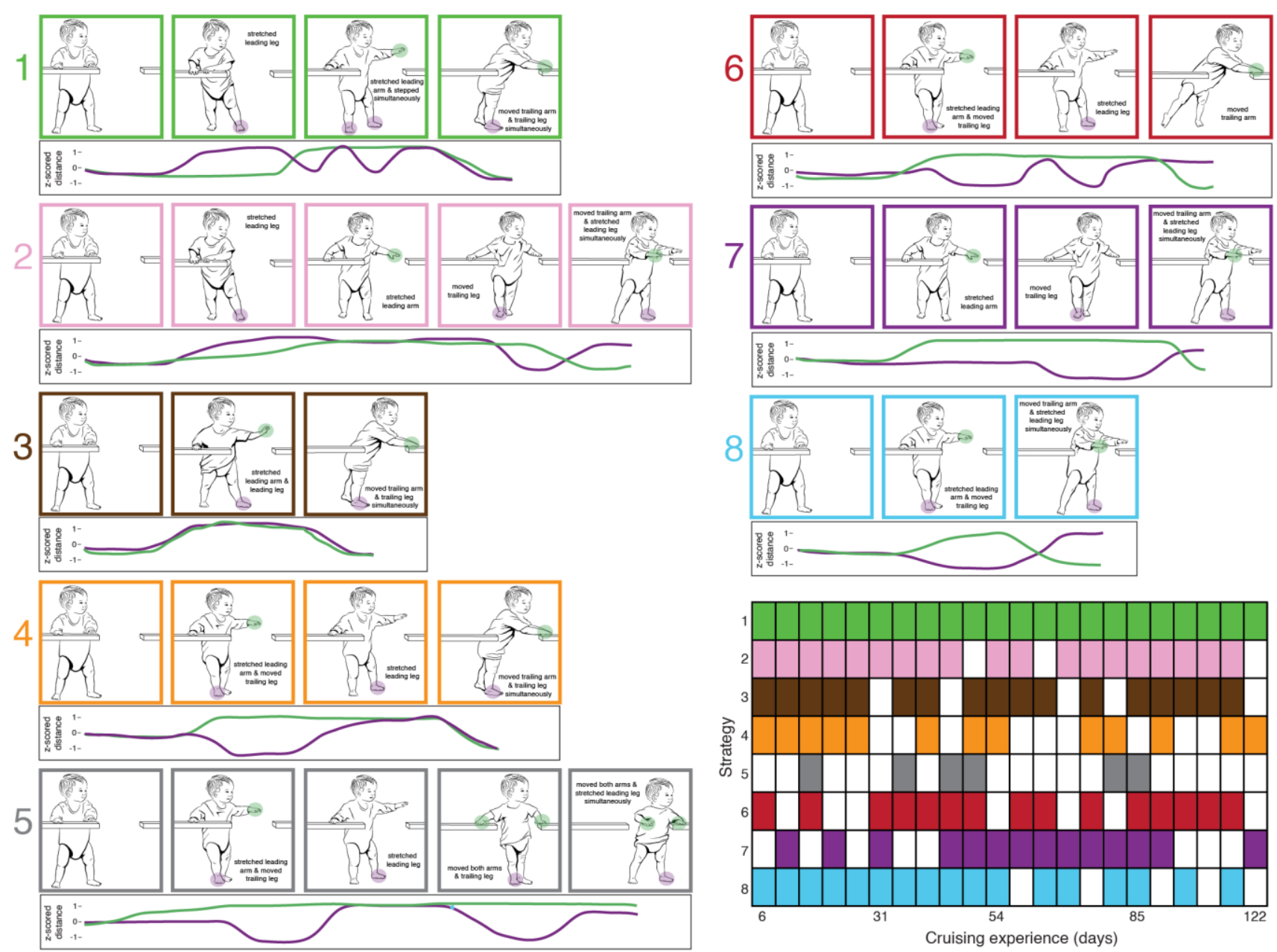

Figure 6. Experiment 2: Drawings of the eight strategies revealed by the cluster analysis (each strategy is color-coded throughout the figure). Left box for each strategy depicts the start of the trial, and each subsequent box represents a change in arm and/or leg movements. Green and purple circles indicate which limbs moved, and the text describes the change in limb configuration. Bottom-right panel shows summary of the coordination strategies for each infant. 

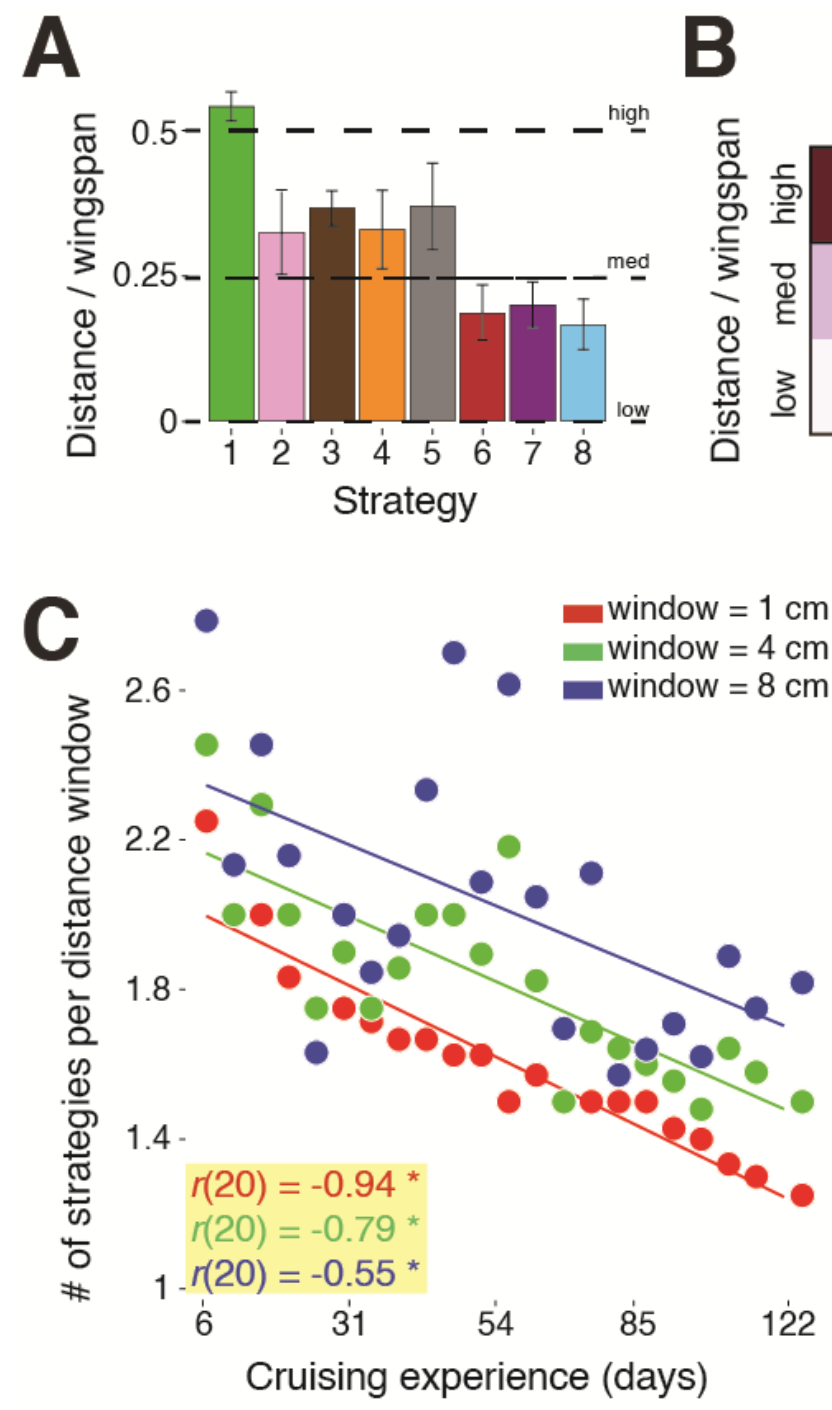
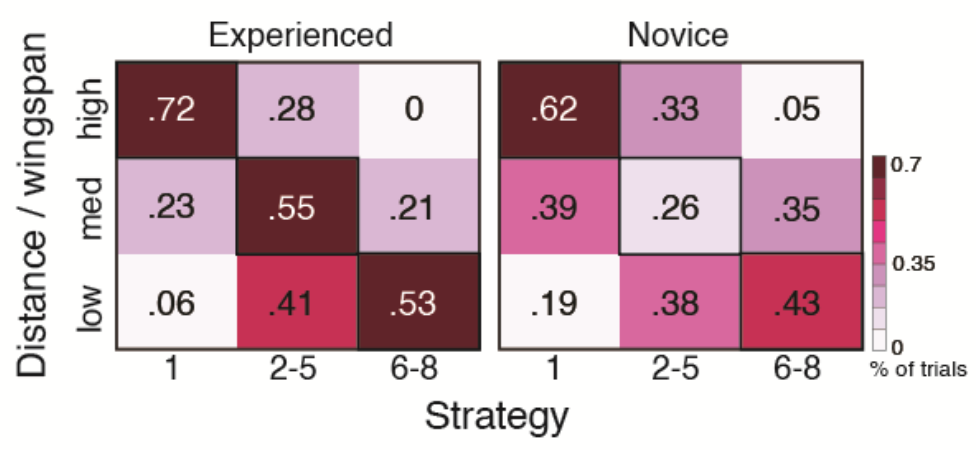

$2.6-$
Strategy

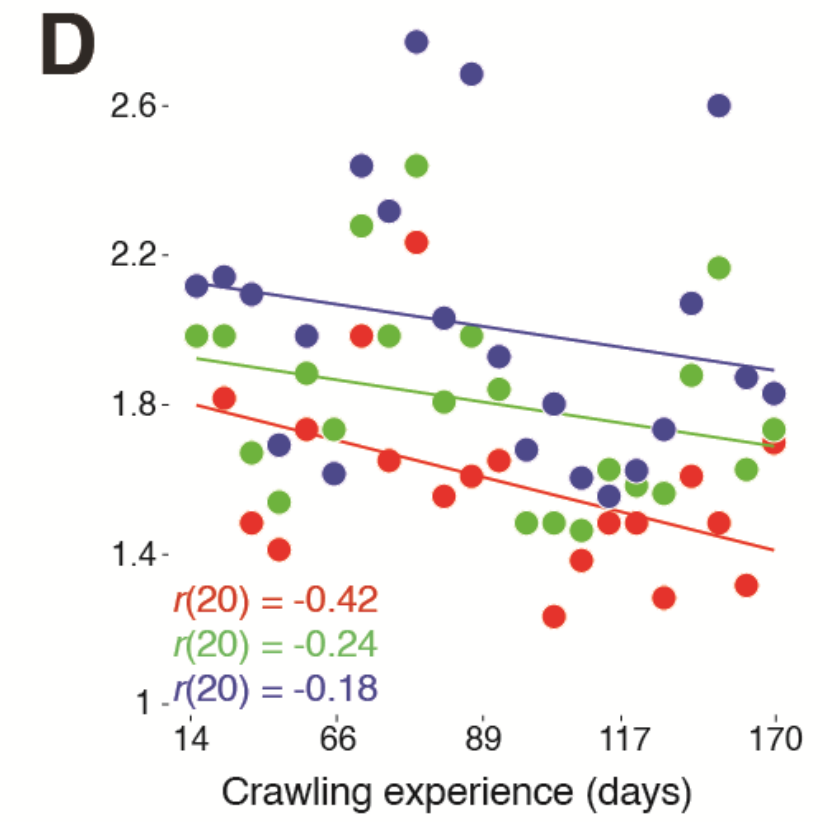

Figure 7. Experiment 2: Changes in coordination strategies with experience. (A) Average ratio between infant wingspan and handrail distance for each strategy. (B) Differences between experienced and novice cruisers in when they used each strategy for each wingspan-distance ratio. (C) Switching index (see STAR Methods) decreased with cruising experience using window sizes of 2,4 , and $8 \mathrm{~cm}$, whereas (D) the switching index did not significantly decrease with crawling experience for any window size. Significant correlations are highlighted in yellow $(p s<.05)$. 


\section{Supplemental Information}

\section{Experiment 2: Variations in Distance between Handrails}

Although the distance between handrails could vary between $0-90 \mathrm{~cm}$, the largest distance any infant cruised successfully was $68 \mathrm{~cm}$ (in most cases, infants crawled across the larger distances rather than avoiding; in a few cases, infants tried to cruise and fell). Half of the

trials, $51.1 \%$, had a $16-\mathrm{cm}$ distance between handrails because we had to repeat relatively easy trials to keep infants motivated. Thus, the number of successful trials and the distribution of handrail distances varied across infants (range $=10$ to 20 trials per infant, $M=15.04$ ). We focused on the portion of the video when infants cruised from one handrail to the other to enable comparisons across distances and infants (see STAR Methods and

\section{https://nyu.databrary.org/volume/1116).}

\section{Replication of Experiment 1: Infants Use Anti-Phase Strategy to Cruise Over a Continuous Handrail}

To replicate Experiment 1 for the dataset in Experiment 2, we used similar computervision algorithms to detect infants' movements and then represented arm and leg movement time series for each trial. For the initial trials in which infants cruised over a solid handrail, we selected the first sequence with 8 consecutive leg steps. For 21 infants, we used Pearson correlations between arm and leg distance for each time series at each video frame to compare interlimb phasing (the algorithm failed to detect cruising on the solid handrail for one infant because of camera movement). As before, infants showed a preferred anti-phase coordination pattern (range $=-.74$ to $.08, M=-.30$ ), and infants with more cruising experience showed larger negative correlations, $r(19)=-.46, p<.04$. 
Table S1. Study 1. Correlations between cruising performance and body dimensions.

\begin{tabular}{|c|c|c|c|c|c|c|c|c|}
\hline \multirow[b]{2}{*}{ Measure } & \multicolumn{2}{|c|}{ Height } & \multicolumn{2}{|c|}{ Weight } & \multicolumn{2}{|c|}{ Leg length } & \multicolumn{2}{|c|}{ Wingspan } \\
\hline & $r(22)$ & $p$ & $r(22)$ & $p$ & $r(22)$ & $p$ & $r(22)$ & $p$ \\
\hline Time to cruise $69 \mathrm{~cm}$ & .16 & .45 & .06 & .78 & -.38 & .06 & -.01 & .96 \\
\hline Number of leg steps & -.08 & .71 & .00 & .99 & .12 & .57 & .00 & .99 \\
\hline Number of arm steps & -.04 & .85 & .09 & .67 & .04 & .85 & -.00 & .99 \\
\hline Variability in leg steps & -.22 & .30 & -.15 & .48 & .20 & .34 & -.08 & .71 \\
\hline Variability in arm steps & -.06 & .78 & -.03 & .88 & .30 & .15 & .02 & .92 \\
\hline Arm-leg cross-correlation & .28 & .20 & -.09 & .69 & .03 & .89 & -.12 & .59 \\
\hline Arm-leg correlation & -.2 & .34 & -.04 & .85 & .26 & .21 & .04 & .85 \\
\hline Arm-leg PLV & -.36 & .08 & .31 & .14 & .00 & .96 & .06 & .78 \\
\hline
\end{tabular}




\section{Supplemental Figures}
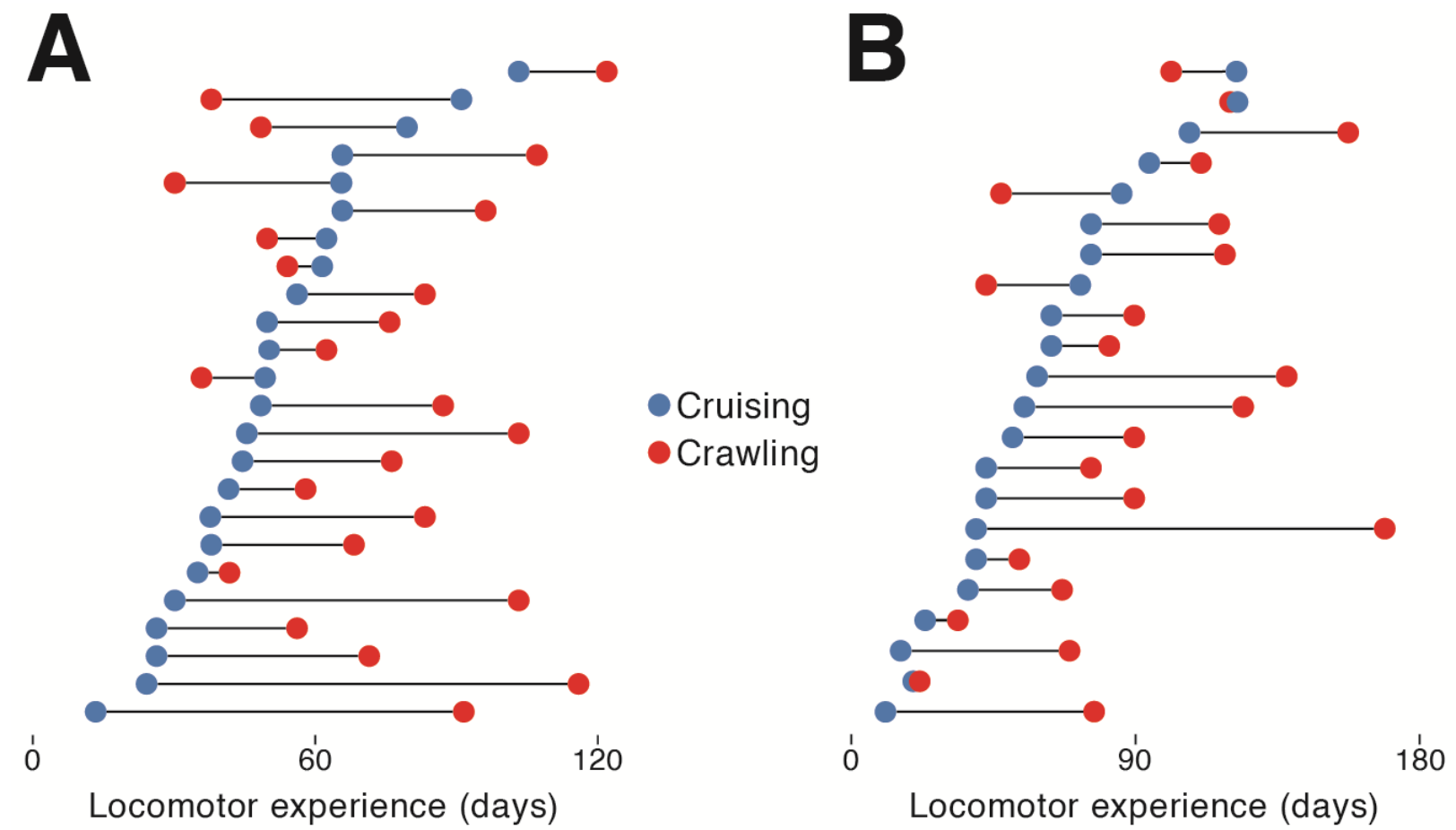

Figure S1. Locomotor experience in days. (A) Experiment 1. Most infants (75\%) had more crawling (red symbols) than cruising experience (blue symbols). (B) Experiment 2. Most infants had more crawling (red symbols) than cruising experience (blue symbols). 


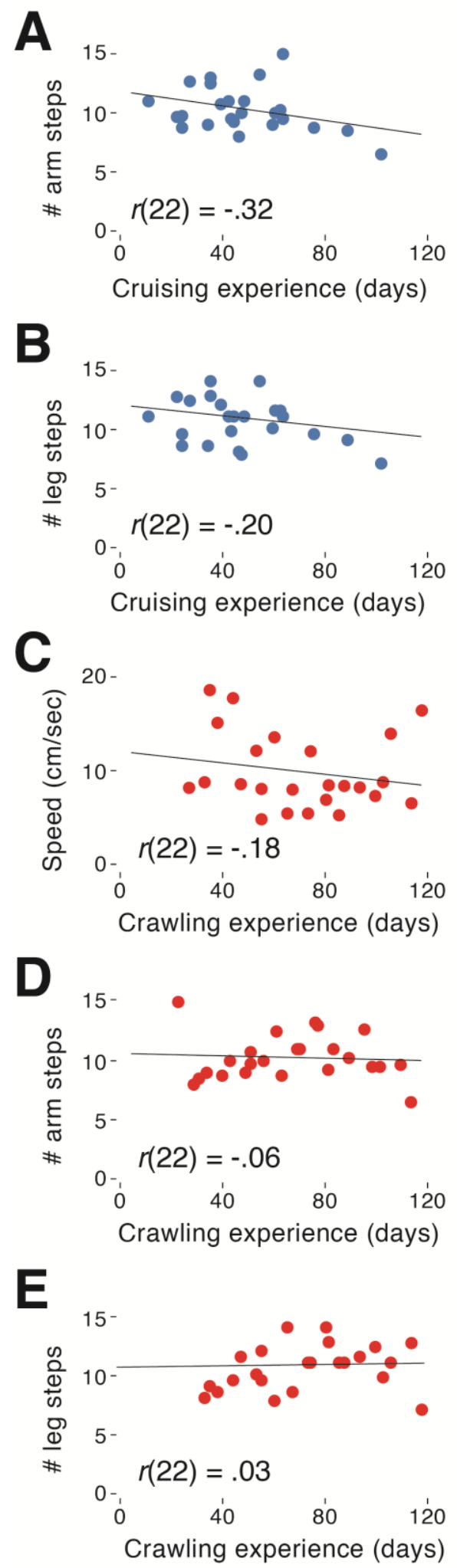

Figure S2. Experiment 1 results: Non-significant correlations between locomotor experience and measures of cruising skill. (A-B) Number of arm and leg steps were not correlated with cruising experience. (C-E) Crawling experience did not predict speed or number of arm or leg steps. 


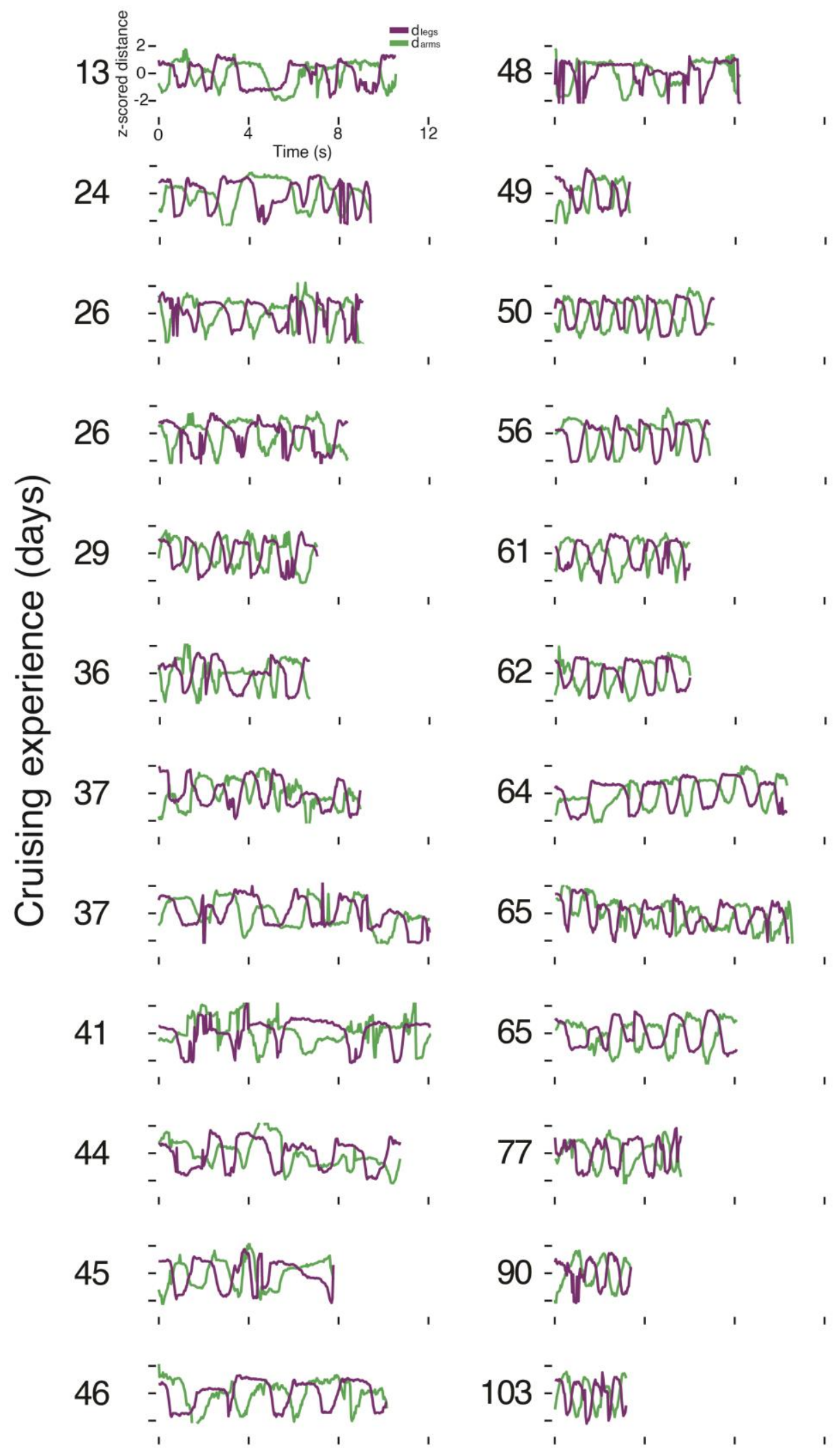

Figure S3. Arm and leg movement time series in one representative trial from each infant, sorted from the least to most experienced cruiser. 

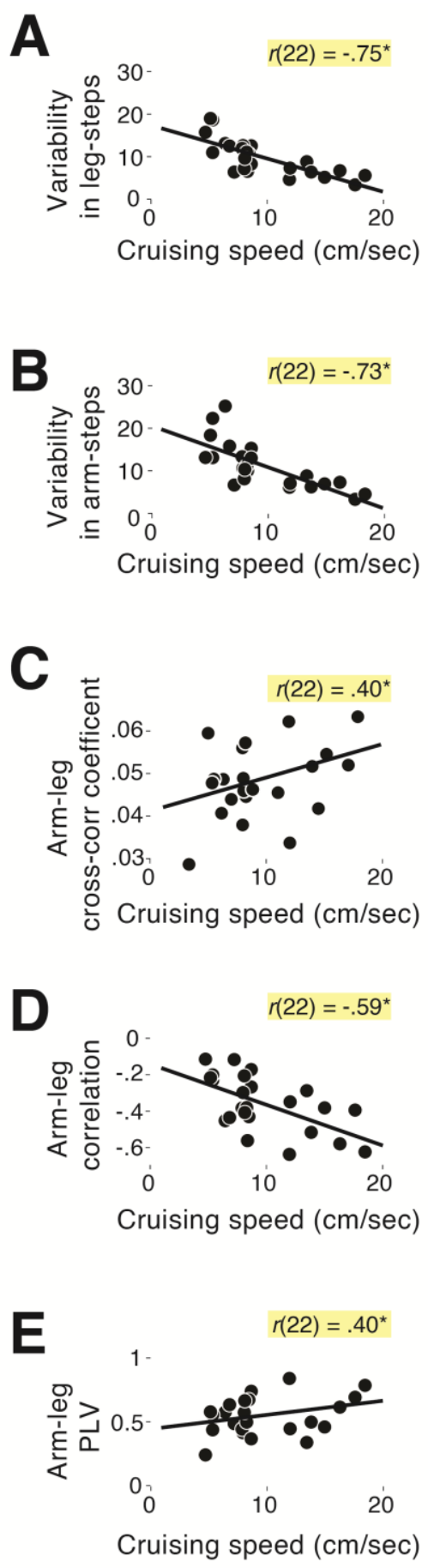

Figure S4. Experiment 1: Scatterplots showing correlations between cruising speed and five measures of interlimb coordination. Significant correlations are highlighted in yellow $(p s<.05)$. (A) Variability in leg movements ( $S D$ of step duration) decreased with speed. (B) Variability in arm movements, like leg movements, decreased with speed. (C) Similarity (maximal crosscorrelation coefficient) between arm and leg movement time series increased with speed. (D) The negative correlations between arm and leg movements (indicating a precise anti-phase coordination pattern) increased with speed. (E) The phase-locking value (see STAR Methods) was positively correlated with speed. 


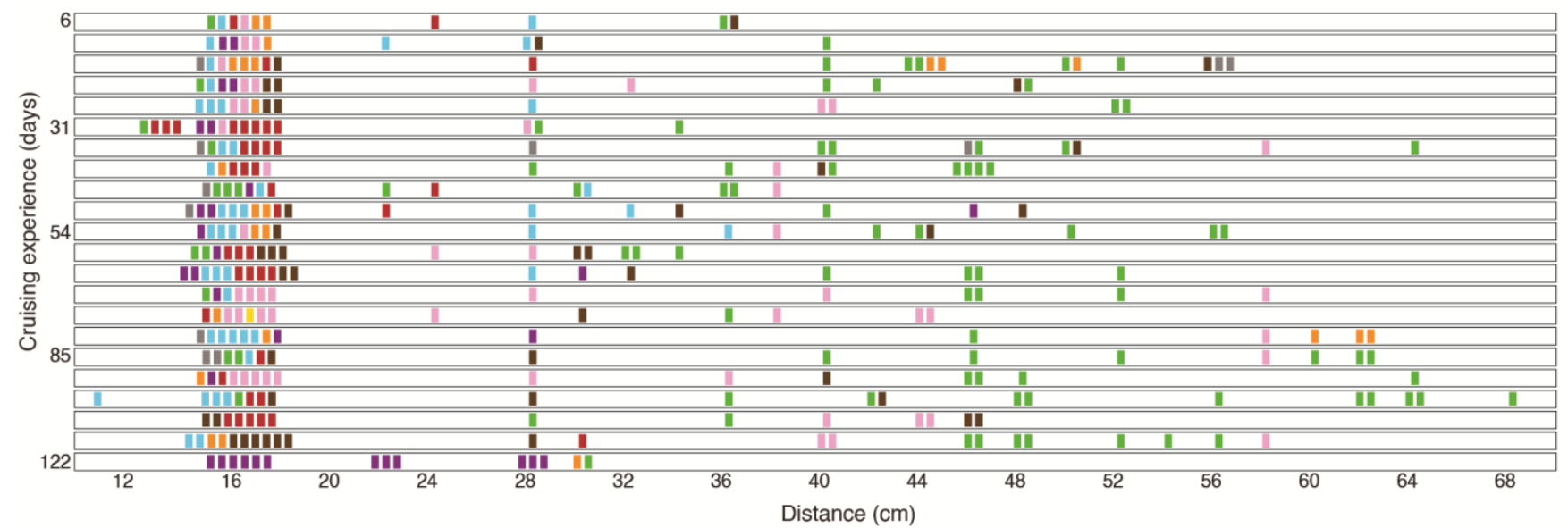

Figure S5. Frequency of each strategy for each infant (rows) and distance. Infants are ordered by cruising experience. Each colored box indicates one trial. 

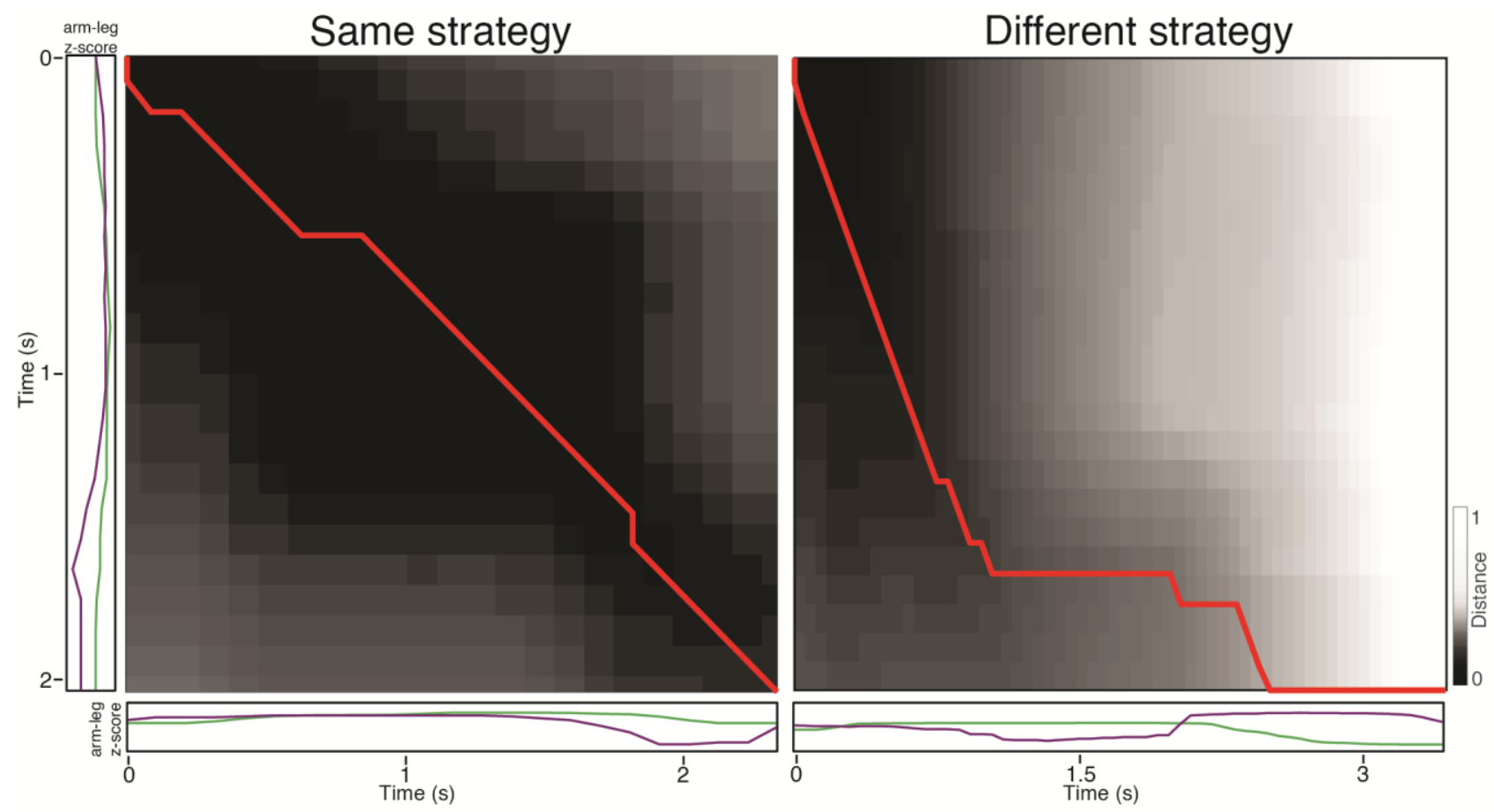

Figure S6. Dynamic time warping analysis in Experiment 2. Example of 2D dynamic time warping analysis, comparing the movement time series from one infant to the movement time series from a second infant with high similarity (left panel) and low similarity (right panel) between their coordination patterns. We constructed a warping matrix and searched for the optimal warping path between the two movement time series, shown by the red line. Similarity was based on the length of the optimal warping path normalized by the duration of the session. Thus, a red line that is close to the diagonal indicates higher similarity than that of an offdiagonal line. 